\title{
SWIFT/BAT AGN2 reveal broad emission lines in the NIR: the first virial measure of their black hole masses
}

\section{Francesca Onori* ${ }^{a}$ Fabio La Franca $^{a}$, Federica Ricci ${ }^{a}$, Marcella Brusa ${ }^{b}$, Eleonora Sani $^{c}$, Roberto Maiolino ${ }^{d}$, Stefano Bianchi ${ }^{a}$, Angela Bongiorno $^{e}$, Fabrizio Fiore $^{e}$, Alessandro Marconi ${ }^{f}$ and Cristian Vignali $^{b}$.}

${ }^{a}$ Dip. di Matematica e Fisica, Università degli Studi "Roma Tre”, via della Vasca Navale 84, 00146, Roma, Italy

${ }^{b}$ Dip. di Fisica e Astronomia, Università di Bologna, viale Berti Pichat 6/2, 40127, Bologna, Italy

${ }^{c}$ INAF - Osservatorio Astrofisico di Arcetri, largo E. Fermi 2, 50125, Firenze, Italy

${ }^{d}$ Cavendish Lab. - University of Cambridge, $19 \mathrm{~J}$. J. Thomson Ave., Cambridge CB3 OHE, UK

${ }^{e}$ INAF - Osservatorio Astronomico di Roma, via Frascati 33, 00044 Monte Porzio Catone, Italy

${ }^{f}$ Dip. di Fisica e Astronomia, Università di Firenze, largo E. Fermi 2, 50125 Firenze, Italy

E-mail: onori, lafranca, riccif, bianchiefis.uniroma3.it,

marcella.brusa3, cristian.vignali@unibo.it, sani@arcetri.astro.it,

r.maiolino@mrao.cam.ac.uk, alessandro.marconi@unifi.it,

angela.bongiorno, fabrizio.fioredoa-roma.inaf.it

In the last decade, using single epoch (SE) virial based techniques in the optical band, it has been possible to measure the Active Galactic Nuclei (AGN) black hole (BH) mass on large type 1 AGN (AGN1) samples and therefore to estimate the supermassive BH mass function (SMBHMF). However these measurements cannot be applied on those obscured type 2 (AGN2) where the nuclear component does not dominate in the optical. The lack of knowledge of BH masses for AGN2 may limit our studies and understanding of AGN/galaxy evolution.

We here present new results from a systematic study carried out using near infrared (NIR) spectroscopy (ISAAC and Xshooter at VLT and LUCI at LBT) of 41 AGN2, drawn from the SWIFT/BAT 70-month hard X-ray survey. We derived a new virial relation able to estimate the $\mathrm{BH}$ mass using the broad component of the $\mathrm{Pa} \beta$ emission line and the AGN hard X-ray luminosity. We have revealed the $\mathrm{Pa} \beta$ broad component in 13 out of 41 AGN2. Thanks to the above relation, we have been able to directly measure their BH masses, finding that AGN2 show, on average, low BH masses $\left(<10^{7} \mathrm{M}_{\odot}\right), 0.8$ dex smaller than those of AGN1 with the same intrinsic hard X-ray luminosity.

Swift: 10 Years of Discovery

2-5 December 2014

La Sapienza University, Rome, Italy

\footnotetext{
* Speaker.
} 


\section{Introduction}

Nowadays there is robust evidence that every galaxy hosts a supermassive black hole (SMBH; $\mathrm{M}_{B H}=10^{6}-10^{9} \mathrm{M}_{\odot}$ ) whose mass scales with the hosting galaxy bulge properties (mass, luminosity and stellar dispersion, [1]; [2]; [3]; [4]). The existence of these scaling relationships implies that the evolution of the galaxy and the growth of SMBHs are intricately tied toghether (AGN/Galaxy co-evolution scenario). In order to obtain a clear picture of the AGN/galaxy co-evolution, it is important to accurately derive the shape and the evolution of both AGN luminosity and SMBHMF in a consistent way. While the complete AGN luminosity function is fairly well measured up to $\mathrm{z} \sim 4$, this is not the case for the SMBHMF. Recently it has been possible to obtain some estimates of the SMBHMF for large samples of type 1 AGN. In this class of AGN the broad line region (BLR) is visible in the rest-frame optical band and this allows the use of virial methods to derive in a direct way the AGN BH mass ([5], [6], [7],[8]). However these kind of measurements are affected by several selection biases against narrow line AGN (AGN2), where the BLR is not visible in the optical because of dust absorption. Moreover, there is growing evidence that AGN1 are not representative of the whole AGN population, having on average larger accretion rates, larger luminosities and larger BH masses with respect to AGN2 ([9], [10], [11]). It is therefore fundamental to directly measure the AGN2 BH mass in order to verify if the two classes of AGN show the same properties.

\section{A new NIR virial relation}

Using a sample of AGN1, whose $\mathrm{BH}$ masses have been previously measured using reverberation mapping techniques (see [12]; [13] and [14]), and following previous studies in which a correlation between the BLR radius and the absorption corrected X-ray luminosity was found ([15]), we have calibrated a new NIR SE BH mass relationship. Our tightest relationship between the BH mass and the virial product has an intrinsic spread of 0.20 dex. This new virial method makes use of the FWHM of the broad component of $\mathrm{Pa} \beta$ emission line and of the hard X-ray luminosity in the 14-195 keV band. Since the X-ray luminosity is less affected by absorption and host galaxy contaminations problems, it is potentially able to derive the BH mass also in AGN2 and low luminosity AGN. We successfully tested this method on one of the lowest BH mass Seyfert 1 known, NGC 4395, finding a new value for its BH mass of $\mathrm{M}_{B H}=1.7_{-0.7}^{+1.3} \times 10^{5} \mathrm{M}_{\odot}$, in agreement with previous estimates. Moreover, thanks to this NIR SE relation we have been able to measure, for the first time, the BH mass for the AGN2 MCG-01-24-12, finding a value of $\mathrm{M}_{B H}=1.5_{-0.6}^{+1.1} \times 10^{7}$ $\mathrm{M}_{\odot}$ (for more details see [16]). Our SE relation and the $\mathrm{Pa} \beta$ emission line fits of NGC 4395 and MCG -01-24-012 are shown in Fig. 1.

\section{The sample and the observations}

The Swift/BAT survey is providing an unprecedented complete and uniform view of the local AGN population, detetected in the 14-195 keV hard X-ray band, almost unbiased against Compton thin $\left(\mathrm{N}_{H}=10^{22}-10^{24} \mathrm{~cm}^{-2}\right) \mathrm{X}$-ray absorbed AGN. The 70-month catalogue ([17]) contains 1171 hard X-ray sources in the 14-195 keV band down to a significance level of $4.8 \sigma$ and, among these, $\sim 600$ are Seyfert galaxies. Counterparts have been found for the $80 \%$ of the sample. 

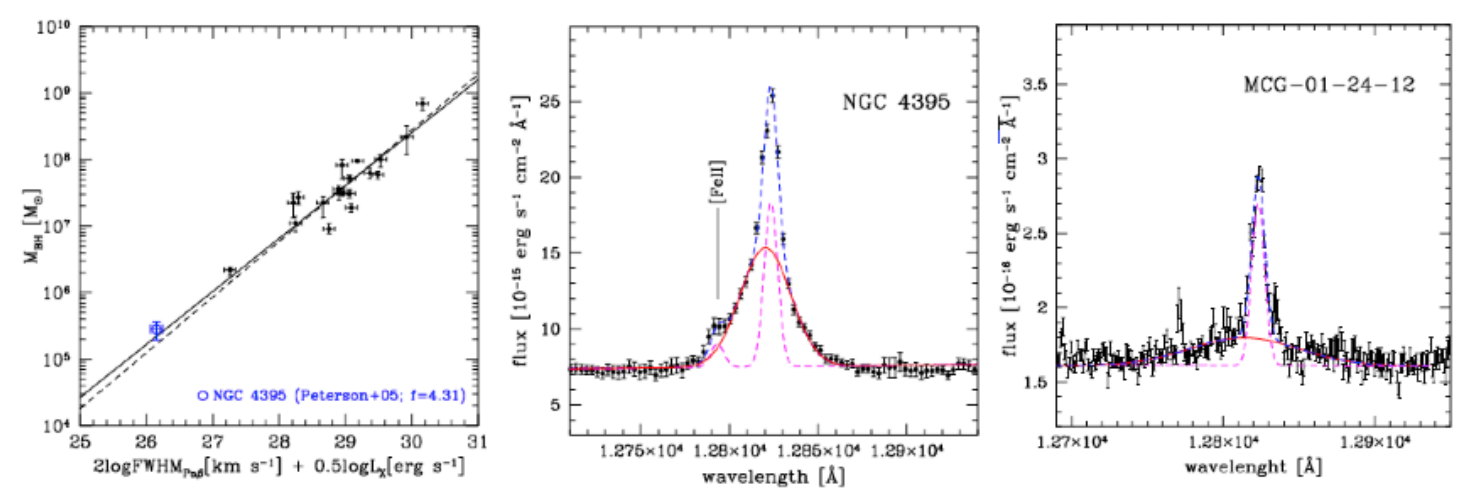

Figure 1: Left: Black Hole masses determined from optical reverberation campaigns (adopting a geometrical factor $\mathrm{f}=4.31$ ) versus the virial product between the hard X-ray luminosity $\mathrm{L}_{X}$ in the $14-195 \mathrm{keV}$ band and the FWHM of the broad component of the $\mathrm{Pa} \beta$ emission line. Center: Rest frame, flux calibrated, LUCI@LBT NIR spectrum of NGC 4395 in proximity of the $\mathrm{Pa} \beta$ emission line. Right: Rest frame, flux calibrated, ISAAC@VLT NIR spectrum of MCG-01-24-012 in proximity of the Pa $\beta$ emission line. The magenta dashed lines show the narrow component of the $\mathrm{Pa} \beta$ and the [FeII] $\lambda 12791$ blended with the $\mathrm{Pa} \beta$, whereas the red solid line is the broad component of $\mathrm{Pa} \beta$. The complete fit is shown by a blue dashed line (see [16] for more details).

In order to study the properties of a local AGN2 sample, we randomly selected from the Swift/BAT 70-month catalogue 41 obscured and intermediate class AGN (AGN2, AGN1.9 and AGN1.8) with $\mathrm{z} \leqslant 0.1$. We observed all these sources in the NIR through dedicated programs with ISAAC/VLT, Xshooter/VLT and LUCI/LBT spectrographs, in order to detect faint broad components in the Paschen emission lines and thus to measure their BH mass using our NIR virial method. In Fig. 2 we show the Hubble diagram of our sample compared with the SWIFT 70-month AGN population.

The observations were performed paying attention to centering the galaxy's nucleus at the best and, when possible, the slit has been rotated in order to include also a telluric star for the $\mathrm{OH}$ absorptions correction. We acquired all the targets using the nodding technique in order to perform a good quality sky correction during the data reduction phase. We also have observed a bright star (O-B or Solar spectral type) within 30 minutes to the target observations, and use it to calibrate the absolute flux and correct for $\mathrm{OH}$ absorptions every time the telluric star was not available. Flats and arcs were taken within one day from the observations. The LUCI targets have been acquired in the $\mathrm{zJ}$ band, using $1 " \times 2.8$ ' slit, corresponding to a $\Delta v=220 \mathrm{~km} / \mathrm{s}$ of spectral resolution. The sources observed with ISAAC spectrograph have been acquired in J band both in Low and Medium resolutions (LR and MR, respectively) spectroscopy and we used a $0.8^{\prime \prime} \times 120^{\prime \prime}$ wide slit, corresponding to a spectral resolution of $\sim 430 \mathrm{~km} / \mathrm{s}$ and $\sim 60 \mathrm{~km} / \mathrm{s}$ for LR and MR mode, respectively. Finally, the targets observed with Xshooter, which covers in a single exposure the spectral range from the UV to the $\mathrm{K}$ band, have been acquired using a $1.0^{\prime \prime} \times 11^{\prime \prime}$ wide slit for the UVB arm and a $0.9^{\prime \prime} \times 11^{\prime \prime}$ for the VIS and NIR arms, corresponding to a $\sim 60 \mathrm{~km} / \mathrm{s}$ velocity resolution. 


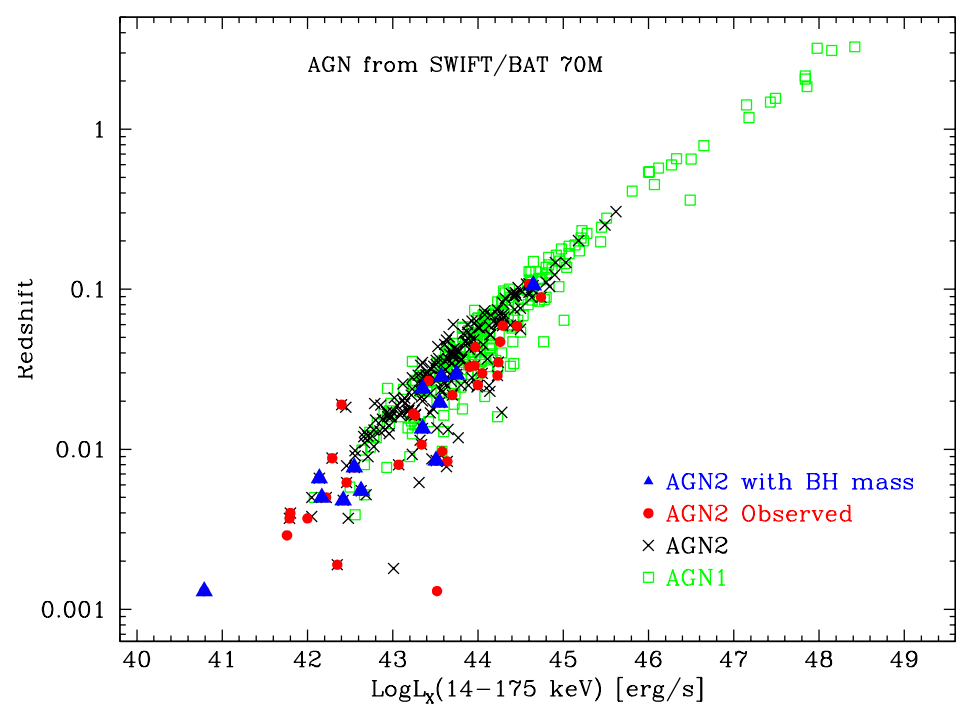

Figure 2: Hubble diagram of the Swift/BAT 70-month sample: AGN1 (green open squares), AGN2 (black crosses), AGN2 observed in the framework of our campaign (red filled dots) and the AGN2 whose BH mass has been derived using our new NIR virial relation based on the broad component of the $\mathrm{Pa} \beta$ line (blue filled triangles).

\section{AGN2 virial BH mass estimation}

We found signatures of broad emission line component in $\mathrm{Pa} \alpha, \mathrm{Pa} \beta$ or $\mathrm{HeI}$ (depending on the redshift of the source) in 13 out of 41 obscured AGN of our sample. The line fit procedure for the broad component FWHM estimate has been performed using the XSPEC software [18]. Despite the low redshift of our targets, the spectra have been converted to the rest frame wavelength. Due to the presence of sistematic errors on the FWHM estimates and following the studies of [12], we have preferred to assume a common uncertainty of $10 \%$ for all the FWHM. All the line fits have been performed paying attention to disentagling the broad component from the narrow one, using, every time it was possible, a forbidden emission line to estimate the width of the NLR contribution. Moreover, following the NIR transitions listed in [12] (Table 4), we have also taken into account the $[\mathrm{FeII}] \lambda 12791 \AA$ in blending with the $\mathrm{Pa} \beta$. In Fig. 1 we show the line fit of the broad $\mathrm{Pa} \beta$ emission line found in the NIR spectra of NGC 4395 and MCG -01-24-012.

\section{Results: the $\mathbf{M}_{B H}-\mathbf{L}_{X}$ plane}

Thanks to our new NIR virial relation and deep NIR observations of a complete sample of obscured and intermediate class AGN, we have been able to derive, for the first time, a virial measure of the BH mass in obscured AGN2. We found a broad component in the Paschen emission line in $\sim 30 \%$ of our AGN sample randomly selected from the Swift/BAT 70-month catalogue, finding that AGN2 show, on average, lower BH masses $\left(<10^{7} \mathrm{M}_{\odot}\right)$ than AGN1. In Figure 3 the $\mathrm{L}_{X}-\mathrm{M}_{B H}$ plane of our 13 AGN2 with virial BH mass estimates (red filled dots) is shown. For comparison, we also plot the distribution of a sample of $\sim 30$ AGN1 (blue empty squares) whose $\mathrm{BH}$ mass estimates have been derived using either reverberation mapping techniques or $\mathrm{M}_{B H}-\mathrm{L}$ 
bulge scaling relations and available in the literature. Our sample of 13 AGN2 results to have, on average, BH masses 0.8 dex smaller than AGN1 having the same intrinsic hard X-ray luminosity. Indeed in the luminosity range $43.2<\log \mathrm{L}_{14-195 \mathrm{keV}}<44 \mathrm{erg} / \mathrm{s}$, AGN1 shows an average BH mass of $\log \mathrm{M}_{B H}=7.6 \pm 0.1 \mathrm{M}_{\odot}$, while our AGN2 show an average BH mass of $\log M_{B H}=6.8 \pm 0.2 \mathrm{M}_{\odot}$. These results suggest that AGN2 populate a different region in the $\mathrm{L}_{X}-\mathrm{M}_{B H}$ plane with respect to the AGN1 population, sampling lower BH masses and luminosities, and for this reason they should be properly taken into account to derive the SMBHMF.

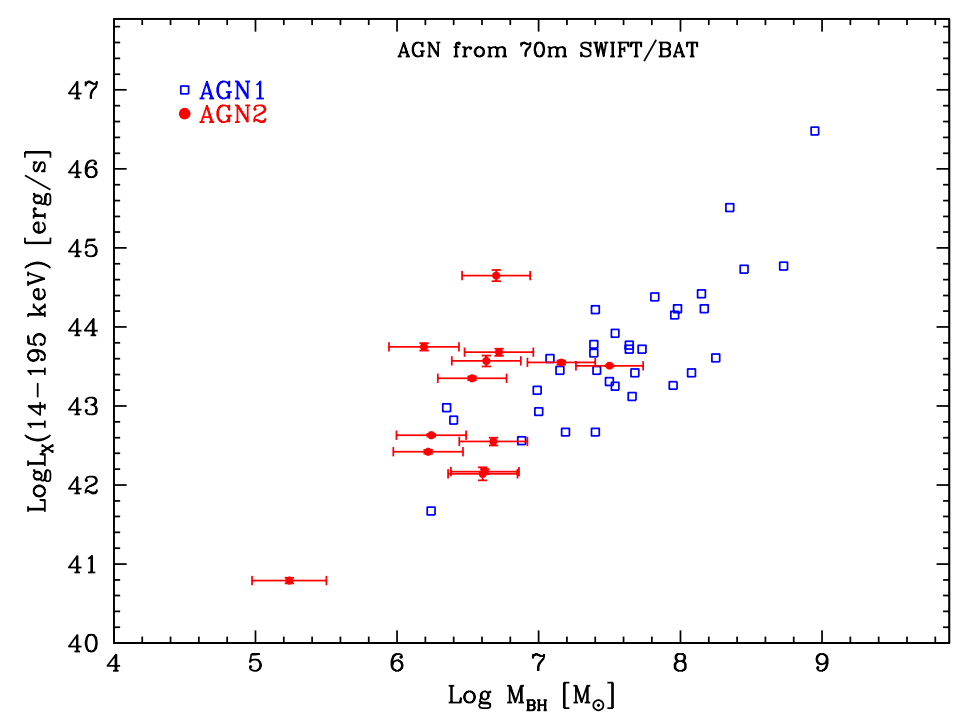

Figure 3: $\mathrm{L}_{X}-\mathrm{M}_{B H}$ plane for our 13 AGN2 with virial estimations of $\mathrm{BH}$ mass (red solid dots). For comparison we also show 34 AGN1 (blue open squares) with BH masses derived from reverberation mapping techniques or the $\mathrm{M}_{B H}-\mathrm{L}$ bulge scaling relations. AGN2 shows $\mathrm{BH}$ masses, on average, 0.8 dex smaller than AGN1 of the same luminosity.

\section{References}

[1] L. Ferrarese and D. Merritt, A Fundamental Relation between Supermassive Black Holes and Their Host Galaxies, ApJ 539 (2000) L9-L12.

[2] K. Gebhardt, R. Bender, G. Bower, A. Dressler, S. M. Faber, A. V. Filippenko, R. Green, C. Grillmair, L. C. Ho, J. Kormendy, T. R. Lauer, J. Magorrian, J. Pinkney, D. Richstone and S. Tremaine $A$ Relationship between Nuclear Black Hole Mass and Galaxy Velocity Dispersion, ApJ 539 (2000) L13-L16.

[3] A. Marconi and L. K. Hunt, The Relation between Black Hole Mass, Bulge Mass, and Near-Infrared Luminosity, ApJL 589 (2003) L21-L24.

[4] E. Sani, A. Marconi, L. K. Hunt and G. Risaliti, The Spitzer/IRAC view of black hole-bulge scaling relations, MNRAS 413 (2011)1479-1494.

[5] J. E. Greene and L. C. Ho, A New Sample of Low-Mass Black Holes in Active Galaxies, ApJ 670 (2007a) 92-104. 
[6] B. C. Kelly, M. Vestergaard and X. Fan, Determining Quasar Black Hole Mass Functions from their Broad Emission Lines: Application to the Bright Quasar Survey, ApJ 692 (2009) 1388-1410.

[7] A. Merloni, A. Bongiorno, M. Bolzonella, M. Brusa, F. Civano, A. Comastri, M. Elvis, F. Fiore and et al., On the Cosmic Evolution of the Scaling Relations Between Black Holes and Their Host Galaxies: Broad-Line Active Galactic Nuclei in the zCOSMOS Survey, ApJ, 708 (2010) 137-157.

[8] A. Bongiorno, R. Maiolino, M. Brusa, A. Marconi, E. Piconcelli, A. Lamastra, M. Cano-Díaz, A. Schulze, B. Magnelli, C. Vignali, F. Fiore, N. Menci, G. Cresci, F. La Franca, A. Merloni, The $M_{B H}-M_{*}$ relation for X-ray-obscured, red QSOs at $1.2<z<2.6$, MNRAS 443 (2014) 2077-2091

[9] F. La Franca, F. Fiore, A. Comastri, G. C. Perola, N. Sacchi, M. Brusa, F. Cocchia, C. Feruglio, G. Matt, C. Vignali, N. Carangelo, P. Ciliegi, A. Lamastra, R. Maiolino, M. Mignoli, S. Molendi and S. Puccetti, The HELLAS2XMM Survey. VII. The Hard X-Ray Luminosity Function of AGNs up to $z=4$ : More Absorbed AGNs at Low Luminosities and High Redshifts, ApJ 635 (2005) 864-879.

[10] L. M. Winter, R. Mushotzky, K. Lewis, S. Veilleux, M. Koss and B. Keeney, Optical and X-ray Properties of the Swift BAT-detected AGN, in proceeding of X-ray Astronomy 2009; Present Status, Multi-Wavelength Approach and Future Perspectives conference, AIPC 1248 (2010) 369-372.

[11] J. Tueller, W. H.Baumgartner, C. B. Markwardt, G. K. Skinner, R. F. Mushotzky, M. Ajello, S. Barthelmy, A. Beardmore, et al., The 22 Month Swift-BAT All-Sky Hard X-ray Survey, ApJS, 186 (2010) 378-405.

[12] H. Landt, M. C. Bentz, M. J. Ward, M. Elvis, M. Peterson, K. T. Korista and M. Karovska, The Near-Infrared Broad Emission Line Region of Active Galactic Nuclei. I. The Observations, ApJS 174 (2008) 282-312.

[13] H. Landt, M. Elvis, M. J. Ward, M. C. Bentz, K. T. Korista and M. Karovska, The near-infrared broad emission line region of active galactic nuclei - II. The 1- $\mu$ m continuum, MNRAS 414 (2011) 218-240.

[14] H. Landt, M. J. Ward, B. M. Peterson, M. C. Bentz, M. Elvis, K. T. Korista and M. Karovska, A near-infrared relationship for estimating black hole masses in active galactic nuclei, MNRAS 432 (2013)113-126.

[15] J. E. Greene, C. E. Hood, A. J. Barth, V. N. Bennert, M. C. Bentz, A. V. Filippenko, E. Gates, M. A. Malkan, T. Treu, J. L. Walsh and J.-H. Woo, The Lick AGN Monitoring Project: Alternate Routes to a Broad-line Region Radius, ApJ 723 (2010) 409-416.

[16] F. La Franca, F. Onori, F. Ricci, E. Sani, M.Brusa, R. Maiolino, S. Bianchi, A. Bongiorno, F. Fiore, A. Marconi and C. Vignali, Extending Virial Black Hole Mass Estimates to Low-Luminosity or Obscured AGN: the cases of NGC 4395 and MCG 01-24-012, MNRAS 449 (2015) 1526-1535.

[17] W. H. Baumgartner, J. Tueller, C. B. Markwardt, G. K. Skinner, S. Barthelmy, R. F. Mushotzky, P. A. Evans and N. Gehrels, The 70 Month Swift-BAT All-sky Hard X-Ray Survey, ApJS 207 (2013) 19.

[18] K. A. Arnaud, Astronomical Data Analysis Software and Systems V, eds. G. Jacoby and J. Barnes, ASP Conf. Series volume, 101 (1996) 17. 